\title{
Bronchioloalveolar carcinoma with metastasis to the pituitary gland: a case report
}

\author{
Gilbert Lau, Soo Yong Tan, Gilbert Chiang, Wee Teng Poh
}

\begin{abstract}
An unusual case of metastatic bronchioloalveolar carcinoma of the lung presented as a pituitary tumour in a young adult Chinese female, who subsequently died after having undergone trans-spheoidal resection. Metastatic cancers of the pituitary are uncommon even in necropsy series and rarely give rise to clinical symptoms. This case draws attention to the fact that, although uncommon, pituitary metastases have been noted with increasing frequency and their distinction from primary pituitary tumours is often difficult. A metastatic pituitary tumour may be the initial presentation of an unknown primary malignancy, wherein the metastatic deposits may also be limited to the pituitary gland. Clinicians and pathologists alike should consider a metastatic lesion in the differential diagnosis of a non-functioning pituitary tumour. (F Clin Pathol 1998;51:931-934)
\end{abstract}

Keywords: bronchioloalveolar carcinoma; pituitary metastasis; perioperative death

\section{Case history}

A 36 year old Chinese woman, who presented with recurrent headaches and diplopia, was initially diagnosed as having migraine and was treated for this. Her past history included a left hemithyroidectomy for nodular goitre with lymphocytic thyroiditis approximately three years before. Computed tomography of the brain, which was done during her current hospital admission, was apparently normal and she was discharged about a week later.

However, she was readmitted barely 10 days later, having had two episodes of what was described as near collapse. After a few days, she showed clinical signs of bilateral abducens nerve palsy and early right papilloedema. Magnetic resonance imaging of the brain suggested the presence of a tumour of the pituitary gland, with suprasellar extension. She then underwent trans-sphenoidal exploration of the pituitary fossa, and an unusually vascular tumour was resected. Subsequent histological examination showed features consistent with a metastatic carcinoma (see below). Interestingly, a preoperative chest radiograph had shown a left hilar mass lesion, but this was thought to be unrelated to the pituitary tumour at that time. Her preoperative plasma electrolyte profile was noted to have been normal.

Postoperatively, the patient was initially in a stable condition. There was no evidence of any leakage of cerebrospinal fluid or of meningitis. Perioperative antibiotic prophylaxis and steroid cover were given and early electrolytic distur- bances caused by transient diabetes insipidus were corrected. Unexpectedly, on the second postoperative day, she suddenly collapsed while dressing herself. Computed tomography of the brain showed evidence of cerebral oedema, without haemorrhage. Despite intensive medical support, which included broad spectrum antibiotics, mannitol infusion, and continued steroid cover, she subsequently died and a coroner's necropsy was carried out.

\section{Necropsy findings}

During necropsy examination, both lungs were found to be markedly consolidated, oedematous, and congested. The lingula of the left lung contained a yellowish subpleural tumour, measuring $2.3 \mathrm{~cm}$ in diameter, with invasive margins and a hard consistency. The subcarinal and the left hilar lymph nodes were enlarged and matted, with a fleshy appearance upon sectioning. The pulmonary vasculature was fully patent, with no evidence of thromboembolism.

There were some blood clots within the sphenoidal sinus, associated with minimal subdural and subarachnoid haemorrhage at the base of the brain, over and around the pituitary fossa, consistent with trans-sphenoidal surgery. No other significant meningeal haemorrhage was found. However, the brain showed cerebral oedema and bilateral uncal grooving, together with diffuse reddish cortical discoloration and early liquefaction. Serial sections of the brain, after formalin fixation, showed blurring of the junction between the grey and white matter, as well as extensive disintegration of the deep grey nuclei, while the pons showed multiple petechial haemorrhages within the tegmentum. No intracerebral or intraventricular haemorrhage was found.

Apart from evidence of a previous left hemithyroidectomy and a small intramural uterine fibroid, there was no other significant gross pathology.

\section{Histopathology}

The lung tumour showed the features of a well differentiated bronchioloalveolar tumour (fig 1), comprising mainly single layers of columnar epithelium, with intranuclear inclusions, lining the alveolar spaces and respiratory bronchioles, with a papillary appearance in some areas. The intervening fibrous stroma was well preserved and the periphery of the tumour was sharply demarcated from the non-neoplastic lung tissue. The malignant cells stained positively for mucin (PAS positive, diastase resistant) and for $\alpha$-1-antitrypsin. The subcarinal and left hilar lymph nodes were found to contain metastases. 


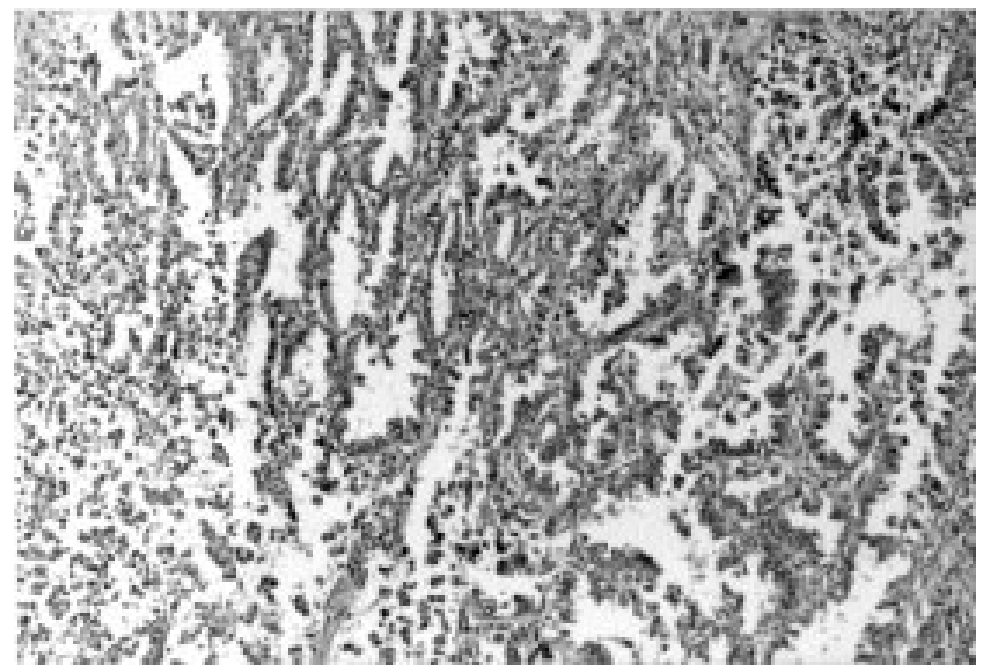

Figure 1 Bronchioloalveolar carcinoma showing malignant columnar epithelial cells lining alveolar walls with preservation of pulmonary architecture $(\times 80)$.

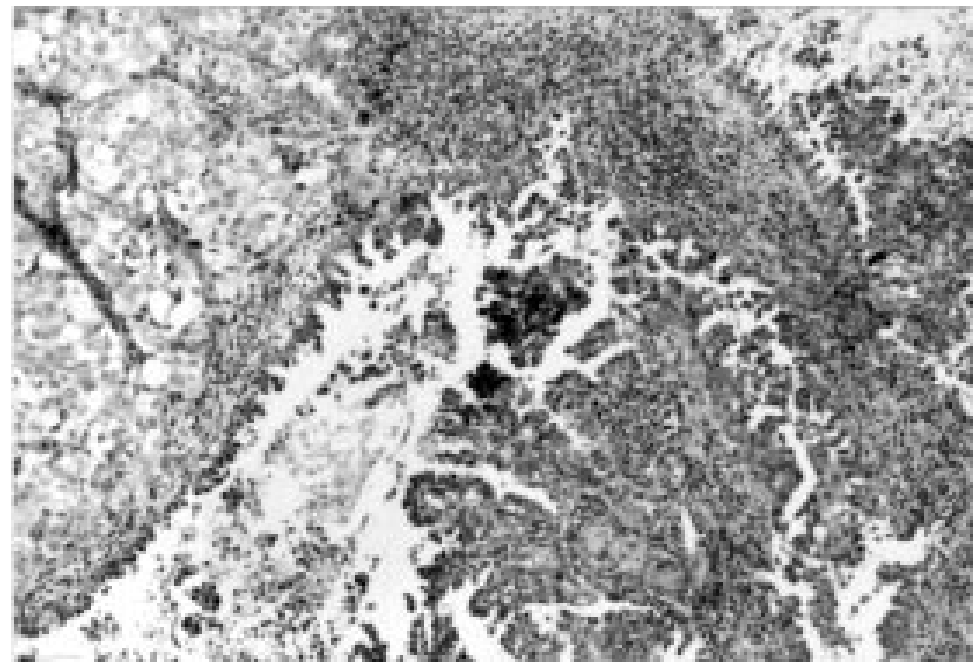

Figure 2 Pituitary tumour comprising papillary fronds lined by tall columnar epithelial cells. Residual pituitary gland is present at one corner of the photomicrograph $(\times 80)$.

These histological features were similar to those of the pituitary tumour (fig 2), consisting of papillary fronds with central fibrovascular cores lined by tall columnar cells, showing moderate nuclear pleomorphism, intranuclear inclusions, and abundant clear to granular cytoplasm. Many tumour cells also presented a hobnail appearance with apical snouting and PAS positive cytoplasmic granules. Immunohistochemistry was performed, with positive results for cytokeratin and epithelial membrane antigen, while stains for thyroglobulin, vimentin, and pituitary hormones were negative.

Transmission electron microscopy of the postmortem specimen showed columnar tumour epithelial cells with well formed desmosomes and the presence of microvilli. Unfortunately, other diagnostic features could not be demonstrated because of autolysis.

Examination of the thyroid gland and a review of the earlier thyroid biopsies and surgical pathology showed no evidence of malignancy, the predominant histological features being a combination of circumscribed proliferation of thyroid follicles of varying sizes, and lymphocytic thyroiditis, associated with occasional germinal centres.

In addition, the brain showed evidence of hypoxic-ischaemic damage, ranging from ischaemic to homogenising cell change and patchy neuronal necrosis, associated with focal neuronal loss in the Sommer's sectors of the hippocampi and almost complete disappearance of the Purkinje cells of the cerebellar cortex, these being accompanied by astrocytosis. Extensive liquefactive necrosis and perivascular haemorrhage was evident in the brain stem. No evidence of meningitis or encephalitis was found.

\section{Cause of death}

Given the rather complex clinicopathological features of this case, the cause of death was eventually certified as being primarily due to cerebral oedema resulting from a metastatic pituitary adenocarcinoma, exacerbated by transsphenoidal resection, with bronchopneumonia as a contributory cause. It is likely that, preoperatively, the pituitary tumour had resulted in some increase in intracranial pressure, together with some degree of vasogenic oedema. This might subsequently have been aggravated by the transient impairment of the blood-brain barrier that almost inevitably follows such surgery, ultimately leading to the patient's demise. Alternatively, subarachnoid haemorrhage, albeit of a limited extent, in and around the circle of Willis, might have predisposed to vasospasm and consequent ischaemic injury to the brain, particularly to the brain stem, with resultant cytotoxic oedema. There was no conclusive evidence that the cerebral oedema was caused by excessive fluid infusion arising from treatment for postoperative diabetes insipidus.

\section{Discussion}

Metastatic tumours are said to constitute $20-40 \%$ of all intracranial tumours, ${ }^{1}$ although in the absence of necropsy examinations of all patients dying from cancer, it is not possible to be precise about the actual incidence or prevalence of metastases to the central nervous system. Carcinomas of the lung, breast, bladder, prostate, ovary, and placenta, as well as melanoma, are the main tumours which give rise to intracranial and spinal metastases, ${ }^{12}$ while metastatic sarcomas are much less common. Metastatic deposits may reside in the brain parenchyma, the cranial and spinal meninges, the skull, and the vertebral column.

Intracranial metastases are known to give rise to a variety of symptoms depending on the site and multiplicity of the lesions. These mainly occur as discrete, often spherical lesions which may induce epilepsy, or present with effects of increased intracranial pressure such as headache, papilloedema, vomiting, and alteration in consciousness. Apart from metastatic deposits in the brain matter, they may spread diffusely through the cerebrospinal fluid, involving the surface of the brain and spinal cord (carcinomatous meningitis) or uncommonly occur in the dura. ${ }^{3}$ Cerebral metastases are often multiple and are distrib- 
uted randomly throughout the cerebral hemispheres and cerebellum.

METASTATIC TUMOURS OF PITUITARY GLAND

Secondary tumours of the pituitary gland are said to be infrequently found at necropsies ${ }^{45}$ and are very rarely recognised clinically. Studies on necropsy material indicate that secondary tumour deposits occur in the hypophysis in about $1-3 \%$ of all cancer patients. ${ }^{6-8}$ In a necropsy series from Memorial Sloan-Kettering Cancer Center, ${ }^{9}$ pituitary metastases were found in $3.6 \%$ of cases and pituitary adenomas in $1.8 \%$. Breast and lung were by far the most frequent primary sites in women and men, respectively. ${ }^{8}$ As noted in this case, metastatic tumours contain abundant vascular networks evidenced by frequent blush on cerebral angiography and increased bleeding at surgery. ${ }^{4}$

Overt clinical symptoms are uncommon, ${ }^{9}{ }^{10}$ apart from occasional diabetes insipidus in patients with metastases to the posterior lobe. The posterior lobe, with its rich arterial blood supply, can be directly involved by metastases through the systemic circulation and is said to be the first to be affected. ${ }^{10}$ Lateral extension from a metastatic deposit in the posterior lobe to the cavernous sinus is known, giving rise to ptosis and diplopia, in addition to diabetes insipidus. ${ }^{4}$ Aaberg et al found oculomotor palsy in $42 \%$ of patients with metastatic tumours compared with less than $5 \%$ of patients with pituitary adenomas. Of patients with metastatic tumours, 33\% had diabetes insipidus, compared with $1 \%$ who had pituitary adenomas. ${ }^{11}$

Impaired anterior pituitary function is less common. The anterior lobes receive blood primarily from the portal vessels and are said to be secondarily involved by direct extension from the posterior lobe, or by haematogenous spread through the portal vessels. ${ }^{10}$ However, isolated metastases in the anterior lobes have been observed. ${ }^{12}$ Hypopituitarism is said to be extremely rare because a large portion of the anterior lobe has to be replaced by tumour before a decrease in adenohypophyseal hormones becomes clinically manifest. ${ }^{12}$ However, Sioutos et al recently reported panhypopituitarism in $25 \%$ of the patients. ${ }^{13}$ Other symptoms and signs reported were impairment of visual acuity, visual field defect, and headaches. ${ }^{14}$

Histological sections of cerebral metastases often show a sharp demarcation between the tumour and surrounding brain tissue. However, in the case of pituitary metastases presenting as primary pituitary tumours, which are often removed by trans-sphenoidal resection, orientation may be lost and this may present a diagnostic problem. Sections stained for mucus are often used to identify metastatic tumours and also as a guide to the site of the primary tumour. Immunohistochemistry may be helpful in cerebral metastases in that metastatic carcinoma cells do not stain for GFAP and often contain cytokeratins. ${ }^{15}$ In the case of an isolated pituitary metastasis on the other hand, it may not be easy trying to differentiate a non-functioning primary pituitary tumour from a metastatic deposit by immunohistochemistry.
BRONCHIOLOALVEOLAR CARCINOMA

Bronchioloalveolar carcinoma (BAC) is a distinctive subset of pulmonary adenocarcinoma "in which cylindrical tumour cells grow upon the walls of pre-existing alveoli". ${ }^{16}$ These tumours show preservation of the underlying pulmonary architecture and are broadly divided into mucinous and non-mucinous types, the latter predominating. BAC constitutes $1-5 \%$ of lung carcinomas in most series ${ }^{17}$ and may present as lobar consolidation radiographically, as slow growing solitary nodules or as multiple, rapidly developing satellite nodules, with resultant widespread bilateral disease. ${ }^{18-20}$ The average age at presentation is in the sixth decade but younger patients, as in this case, have been reported, even in the second decade. ${ }^{21}{ }^{22}$ The key histological picture of BAC is the growth of cuboidal and columnar tumour cells along intact alveolar walls either in a single layer or forming papillae. Nonmucinous BAC are more often cuboidal with brightly eosinophilic cytoplasm, with large atypical nuclei showing prominent nucleoli and pleomorphism. Clara cell differentiation may be evidenced by apocrine snouting in a hobnail fashion and the presence of PAS positive apical cytoplasmic granules, seen in $90 \%$ of nonmucinous BAC. ${ }^{23}{ }^{24}$ Type II pneumocyte differentiation may be suggested by the presence of cytoplasmic osmiophilic lamellar bodies and positive staining for surfactant apoprotein.

BAC may show haematogenous, lymphatic, and pleural invasion but the distinctive feature of this tumour is the propensity for aerogenous spread within air spaces, leading to descriptions such as "lepidic spread," not dissimilar to the picture of butterflies alighting along the alveolar walls. The overall five year survival is $42.1 \%$, with over $90 \%$ five year survival reported in T1N0M0 tumours. ${ }^{21}$ Higher stage disease and patients with evidence of metastases have a worse prognosis that approaches other forms of lung cancer, as is evident in our case.

PRIMARY VERSUS METASTATIC PITUITARY

TUMOUR

This case presented a diagnostic problem owing to several atypical features. The patient was young without the usual risk factors for lung cancer. The clinical presentation of a pituitary tumour with suprasellar extension and increased intracranial pressure in a young woman rightly led to the clinical impression of a primary pituitary tumour. This clinical suspicion was so strong that, perhaps, the finding of a hilar mass lesion on the chest radiograph was discounted.

As noted before, although metastatic tumours of the pituitary gland and the sellar region are not common, they may radiographically mimic pituitary tumours. ${ }^{4} 132526$ Furthermore, though some clinical features may suggest the presence of a metastasis, the diagnosis is less likely both clinically and radiologically and more common pituitary lesions are generally suspected. ${ }^{527}$ Aaberg et al reported that with respect to differentiating a pituitary adenoma from a metastatic process, 
radiographic examinations were not helpful. ${ }^{11}$ Similarly, Max et al found that radiological evaluation, including polytomography and computed tomography, did not reliably distinguish a metastasis from an adenoma.

The histopathology of the resected tumour also presented problems in interpretation and indeed Aaberg et al noted that histopathological examination was occasionally incorrect in their series. ${ }^{11}$ The surgical excision specimen in this case was obviously an adenocarcinoma with prominent papillary features. A panel of immunohistochemical stains was performed, but apart from strong staining for cytokeratins the rest were equivocal. In particular, stains for pituitary hormones were either negative or weakly positive and the possibility of a non-functioning pituitary carcinoma was considered. The prominence of papillae and occasional intranuclear inclusions also led us to consider papillary thyroid carcinoma and papillary meningioma. However, immunoperoxidase stains for thyroglobulin and vimentin were negative. Whereas epithelial markers such as cytokeratins and epithelial membrane antigen do not assist in differentiating a metastatic adenocarcinoma from a malignant choroid plexus papilloma or papillary meningioma, the presence of vimentin in a meningioma would distinguish it from a carcinoma. ${ }^{28}$

CONCLUSIONS

This case of a bronchioloalveolar carcinoma presenting with an isolated metastatic deposit localised to the pituitary gland is distinctly unusual, although pituitary metastatic tumours have been known to occur in patients with widespread metastatic disease in other organs..$^{512}$ Recently, Sioutos et al also found that, of the patients with metastatic pituitary tumours in their series, metastasis was limited to the pituitary gland only in $50 \%$ of cases. ${ }^{13}$

Increasingly, there have been reports of the pituitary lesions representing the onset symptoms of an otherwise unknown malignancy. ${ }^{5142529}$ Ruelle et al suggest that the diagnosis of pituitary metastases should be more closely considered even in the absence of a known primary tumour. ${ }^{5}$

An isolated pituitary metastasis can closely mimic a pituitary adenoma, which is far more common. Juneau et al drew attention to the fact that metastatic tumour in the pituitary fossa can mimic a non-functioning pituitary adenoma in clinical presentation and imaging, and that rapidly progressive visual loss, extraocular movement palsies, or facial sensory loss may help to distinguish it from a benign adenoma. ${ }^{29}$ Verhelst et al stated that even in the absence of suggestive symptoms such as diabetes insipidus or cranial nerve paralysis, the possibility of metastatic disease in the differential diagnosis of a pituitary mass should always be considered. ${ }^{25}$ The pathologist who is evaluating a suspected non-secretory or nonfunctional adenoma would do well to consider the possibility of a metastatic lesion in the differential diagnosis.

1 Russel DS, Rubinstein LJ. Pathology of tumours of the nervous system, 5th ed. Revised by Rubinstein LJ. London: Arnold, system, 5 th ed.
1989:829-30.

2 Burger PC, Scheithauer BW. Tumors of the nervous system, 3rd series. Fascicle 10. Washington DC: Armed forces Institute of Pathology, 1993:415.

3 Weller RO. Nervous system, muscle and eyes, 3rd ed. Volume . Systemic pathology. London: Churchill Livingstone, 1990:487

4 Nelson PB, Robinson AG, Martinez AJ. Metastatic tumor of the pituitary gland. Neurosurgery 1987;21:941-4.

5 Ruelle A, Palladino M, Andrioli GC. Pituitary metastases as presenting lesions of malignancy. $\mathcal{f}$ Neurosurg Sci 1992;36: 51-4.

6 Hagerstrand I, Schonebeck J. Metastases to the pituitary gland. Acta Pathol Microbiol Scand 1969;75:64.

7 Kovacs, K. Metastatic cancer of pituitary gland. Oncology 1973;27:533.

8 Teears RJ, Silverman EM. Clinicopathologic review of 88 cases of carcinoma metastatic to the pituitary gland. Cancer 1975;36:216.

9 Max MB, Deck MD, Rottenberg DA. Pituitary metastasis: incidence in cancer patients and clinical differentiation from pituitary adenoma. Neurology 1981;31:998-1002.

10 Duchen LW. Metastatic carcinoma in the pituitary gland and hypothalamus. F Pathol Bacteriol 1966;91:347.

11 Aaberg TM, Kay M, Sternau L. Metastatic tumors to the pituitary. Am f Ophthalmol 1995;119:779-85.

12 Bloodworth JMB. Endocrine pathology: general and surgical, 2nd ed. Baltimore: Williams \& Wilkins, 1982:128.

13 Sioutos P, Yen V, Arbit E. Pituitary gland metastases. Ann Surg Oncol 1996;3:94-9.

14 Chiang MF, Brock M, Patt S. Pituitary metastases. Neurochirurgia (Stuttg) 1990;33:127-31.

15 Weller RO. Nervous system, muscle and eyes, 3rd ed. Volume 4. Systemic pathology. London: Churchill Livingstone, 1990:490.

16 Sobin L, Yesner R. Histological typing of lung tumours. International histological classification of tumors, vol 1, 2nd ed. Geneva: World Health Organisation, 1981:25.

17 Colby TV, Koss MN, Travis WD. Tumors of the lower respiratory tract, 3rd series. Fascicle 13. Washington DC: Armed Forces Institute of Pathology, 1994:203.

18 Edwards CW. Alveolar carcinoma: a review. Thorax 1984;39:166-74

19 Greco RJ, Steiner RM, Goldman S, et al. Bronchoalveolar cell carcinoma of the lung. Ann Thorac Surg 1986;41:652-6.

20 Marzano MJ, Deschler T, Mintzer RA. Alveolar cell carcinoma. Chest 1984;86:123-8.

21 Daly RC, Trastek VF, Pairolero PC. Bronchoalveolar carcinoma: factors affecting survival. Ann Thorac Surg 1991;51:368-77.

22 Edgerton F, Rao U, Takita H, et al. Bronchio-alveolar carcinoma. A clinical overview and bibliography. Oncology 1981;38:269-73.

23 Clayton F. Bronchioloalveolar carcinomas. Cell types, patterns of growth, and prognostic correlates. Cancer 1986; 57:1555-64.

24 The spectrum and significance of bronchioloalveolar carcinomas. Pathol Annu 1988;23:361-94.

25 Verhelst J, Vanden Broucke P, Dua G, et al. Pituitary metastasis mimicking a pituitary adenoma. A description of two cases. Acta Clin Belg 1995;50:31-5.

26 Branch CL, Laws ER. Metastatic tumors of the sella turcica masquerading as primary pituitary tumors. F Clin Endocrinol Metab 1987;65:469-74

27 McCormick PC, Post KD, Kandji AD, et al. Metastatic carcinoma to the pituitary gland. Brf Neurosurg 1989;3:71-9.

28 Russel DS, Rubinstein LJ. Pathology of tumours of the nervous system, 5th ed. Revised by Rubinstein LJ. London: Arnold, 1989:836

29 Juneau P, Schoene WC, Black P. Malignant tumors in the pituitary gland. Arch Neurol 1992;49:555-8. 